\title{
BER Performance Analysis of a Concatenated Low Density Parity Check Encoded OFDM System in AWGN and Fading Channels
}

\author{
M. D. Haque ${ }^{1}$, S. E. Ullah ${ }^{2}$, M. M. Rahman ${ }^{3}$, and M. Ahmed ${ }^{2}$ \\ ${ }^{1}$ Department of Computer and Communication Engineering, International Islamic University \\ Chittagong, Chittagong-4203, Bangladesh \\ ${ }^{2}$ Department of Information and Communication Engineering, Rajshahi University, Rajshahi-6205, \\ Bangladesh \\ ${ }^{3}$ Department of Applied Physics, Electronics and Communication Engineering, Islamic University, \\ Kushtia-7003, Bangladesh
}

Received 23 June 2009, accepted in revised form 25 November 2009

\begin{abstract}
In this paper, we investigate the bit error rate (BER) performance of a concatenated low density parity check (LDPC) encoded orthogonal frequency-division multiplexing (OFDM) system on color image transmission. In FEC concatenated channel coding, the OFDM wireless communication system incorporates $1 / 2$-rated convolution encoder under various digital modulations (QPSK, 8PSK, QAM, 8QAM, 16QAM and 32QAM) over an additative white gaussian noise (AWGN) and other fading (Raleigh and Rician) channels. Computer simulation results on BER demonstrate that the LDPC encoded OFDM system outperforms with QAM modulation technique as compared to other digital modulation scheme and the system is highly effective to combat inherent interferences under Rayleigh and Rician fading channels. The transmitted color image is found to have retrieved effectively under noisy and fading situations with implementation of sum-product algorithm, an effective iterative based LDPC decoding scheme. It has also been anticipated that the performance of the communication system degrades with the increasing of noise power.
\end{abstract}

Keywords: Orthogonal frequency division multiplexing (OFDM); Low density parity check (LDPC); Bit error rate (BER).

(C) 2010 JSR Publications. ISSN: 2070-0237 (Print); 2070-0245 (Online). All rights reserved.

DOI: 10.3329/jsr.v2i1.2724 J. Sci. Res. 2 (1), 46-53 (2010)

\section{Introduction}

Orthogonal frequency division multiplexing (OFDM) is a very attractive multi carrier modulation technique and is being implemented to achieve high bit data rate transmission. This technique is well suited to overcome adverse effects in hostile transmission environment. This technique provides a reliable reception of signals affected by multipath

\footnotetext{
${ }^{1}$ Corresponding author: dulal_ape83@yahoo.com
} 
propagation and selective fading and has been used in broadcast media such as European terrestrial digital video broadcasting (DVB-T) and digital audio broadcasting (DAB) and in the IEEE 802.11a (local area network, LAN) and the IEEE 802.16a (metropolitan area network, MAN) standards. OFDM is also being pursued for dedicated short-range communications (DSRC) for road side to vehicle communications. With the advent of next generation (4G) broadband wireless communications, the combination of multipleinput multiple-output (MIMO) wireless technology with orthogonal frequency division multiplexing (OFDM) has been recognized as one of the most promising techniques to achieve high data rate and provide more reliable reception compared with the traditional single antenna system [1, 2].

In OFDM system, the wide signal bandwidth is divided into many narrowband subchannels that are transmitted in parallel. To improve the error rate performance, various types of forward error correction codes such as Convoluional code, Reed-Solomon, Turbo codes, etc are reported to have been applied to OFDM. In recent years, low density parity check (LDPC) codes have attracted much attention particularly in the field of coding theory. LDPC codes were proposed by Gallager in $1962[3,4]$ and the performance is very close to the Shannon limit with practical decoding complexity like Turbo codes. The performance of LDPC codes has been also evaluated on a block fading channel and it has been shown that the LDPC codes achieve a large gain with respect to convolutional codes for large packet length [5]. The LDPC codes are very much effective to improve the error performance of OFDM in multipath environments and such LDPC codes can be decoded by using a probability propagation algorithm known as the sum-product algorithm or belief propagation [6]. In this paper, we evaluate the bit error rate (BER) of an orthogonal frequency division multiplexing system with concatenation of outer low-density paritycheck and inner convolutional channel coding schemes working under each of the six types of digital modulation (QPSK, 8PSK, QAM and 8QAM, 16QAM and 32QAM) in the AWGN, Rayleigh and Rician fading channels.

\section{LDPC Coding}

Low-density parity check (LDPC) is an emerging new technique that gets even more closer to Shannon rate with long code words. LDPC codes are linear block codes showing good block error correcting capability and linear decoding complexity in time. Its use in concatenated channel coding achieves considerable reduction of error probability with less complexity than a single code. A $(n, k)$ LDPC encoder operates on an $m \times n$ sized $H$ matrix where $m=n$ - $k$. It is low density because the number of $1 s$ in each row $w_{r}$ is $« m$ and the number of $1 s$ in each column $w_{c}$ is $" n$. A LDPC is regular if $w_{c}$ is constant for every column and $w_{r}=w_{c}(n / m)$ is also constant for every row. Otherwise it is irregular. In LDPC encoding, the codeword $\left(c_{0}, c_{1}, c_{2}, c_{3}, \ldots, c_{n}\right)$ consists of the message bits $\left(m_{0}, m_{l}\right.$, $\left.m_{2}, \ldots, m_{k}\right)$ and some parity check bits and the equations are derived from $H$ matrix in order to generate parity check bits. The solution in solving the parity check equations can be written as:

$$
H c^{T}=0
$$


where such mathematical manipulation can be performed with a generator matrix $G$. $G$ is found from $H$ with Gaussian elimination which can be written as follows:

$$
H=\left[P^{T}: I\right]
$$

and $G$ is

$$
G=[I: P]
$$

Hence, $c$ codeword is found for message word $x$ as follows $c=x G=[x: x P]$.

The graphical representation for a typical $(8,4)$ LPDC encoding is shown in Fig. 1. The graphical representation utilizes variable nodes ( $v$-nodes) and check nodes ( $c$-nodes). The graph has four $c$-nodes and eight $v$-nodes. The check node $f_{i}$ is connected to $c_{i}$ if $h_{i j}$ of $H$ is a 1 . This is important to understand the decoding. Decoding tries to solve the $(n-k)$ parity check equations of the $H$ matrix. There are several algorithms defined to date and the most common ones are message passing algorithm, belief propagation algorithm and sum-product algorithm [7]. In this paper, we have employed sum-product decoding algorithm as presented in [8].

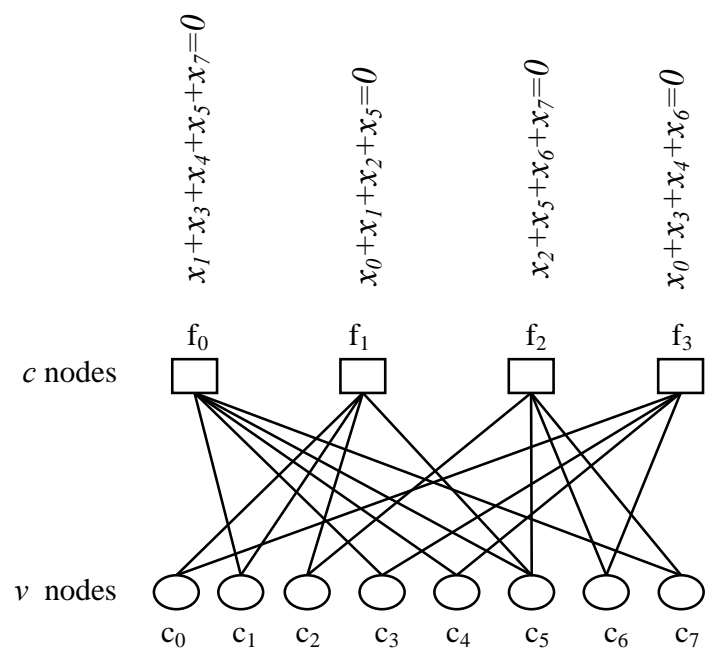

Fig. 1. Graphical representation of a $(8,4)$ LDPC code.

\section{System Description}

We assume that a simulated orthogonal frequency division multiplexing system depicted in Fig. 2 utilizes a concatenated low-density parity-check coding scheme. As an input information source, a non-compressed digital color (RGB) image in Joint Photographic Experts Group (JPEG) format is used. The standard image is discretized into raster of 100 $\times 90$ picture elements and the analog transmission signals RGB are converted into digital image components after analog-to-digital conversion [9]. The digital image samples of each RGB components are multiplexed serially and encoded as a block of $k$ input bits (21600) in the LDPC encoder by a rate $r=k / n$ LDPC code. The encoder output $n$ (43200) coded bits are interleaved and subsequently applied to the $1 / 2$-rated Convolutional encoder 
with constraint length of 7. The eonvolutionally encoded interleaved bits are modulated digitally by phase shift keying (PSK)/ quadrature amplitude modulated (QAM) constellation $\Omega$ into a block of $n / \log _{2}|\Omega|$ complex PSK/QAM modulation symbols [10].

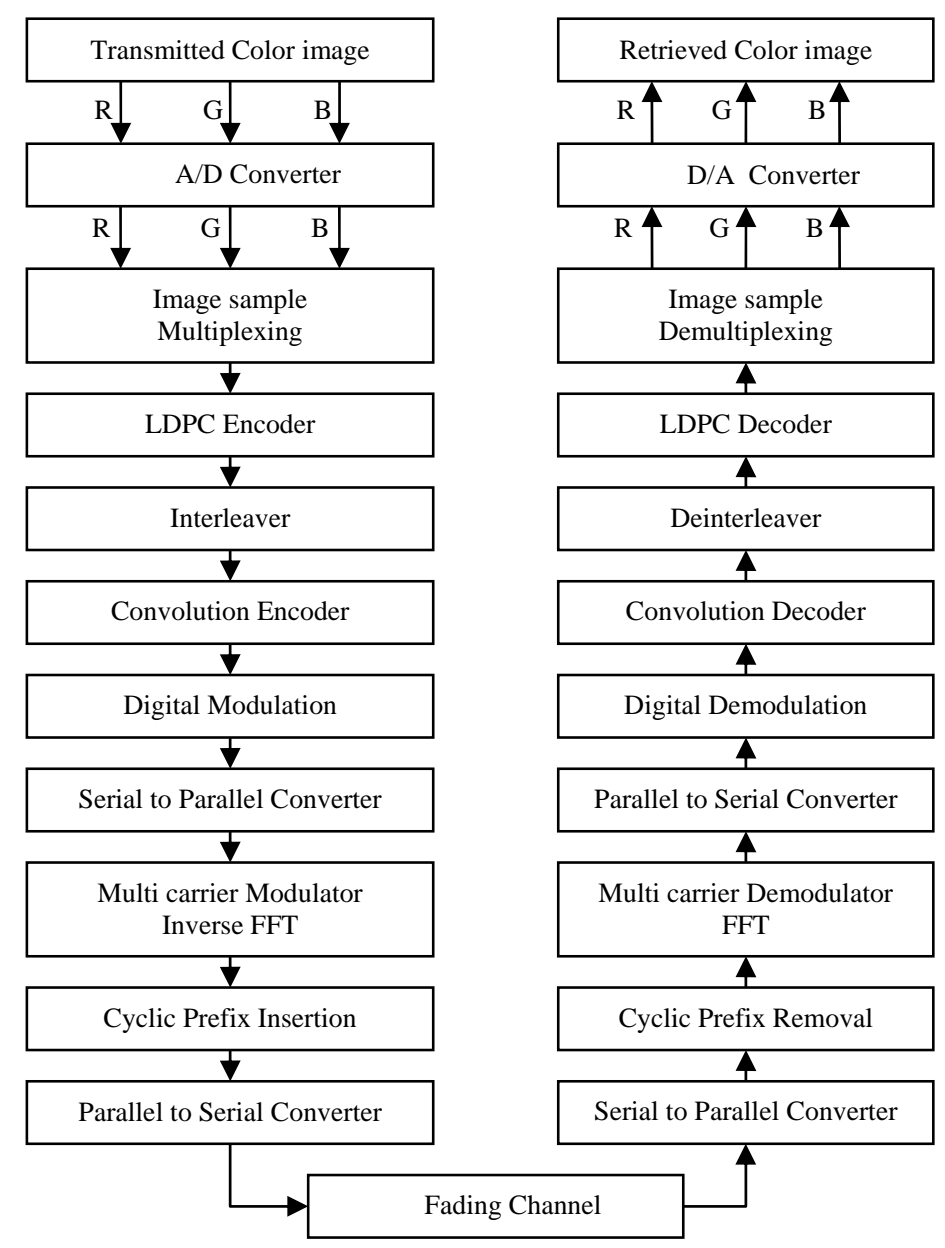

Fig. 2. Block diagram of a concatenated LDPC encoded orthogonal frequency division multiplexing (OFDM) system.

The symbols are then fed into OFDM modulator which performs an IFFT on each OFDM block of length 64 symbols/ sub carriers followed by a parallel to serial conversion. A cyclic prefix (CP) of length $L_{c p}$ containing a copy of the last $L_{c p}$ samples of the parallel to serial converted output of the 64-point IFFT is then prepended. The resulting OFDM symbols of length $64+L_{c p}$ are lunched from the transmit antenna. The CP is essentially a guard interval which serves to eliminate interference between OFDM symbols and turns linear convolution into circular convolution such that the channel is diagonalized by the 
FFT. In the receiver the signals are passed through OFDM demodulators which first discard the CP and then perform a 64-point FFT [11]. The outputs of the OFDM demodulators are finally de-mapped, convolutionally decoded and de-interleaved and then fed into the LDPC decoder to retrieve bits using an iterative sum-product algorithm. The LDPC decoded bit stream is demultiplexed into digital image samples of each RGB components and after analog-to-digital conversion, the transmitted color image is retrieved.

\section{Simulation Results}

Figs. 3 through 5 show the BER performance of a concatenated LDPC encoded OFDM system under six types of digital modulations on both AWGN and fading channels.

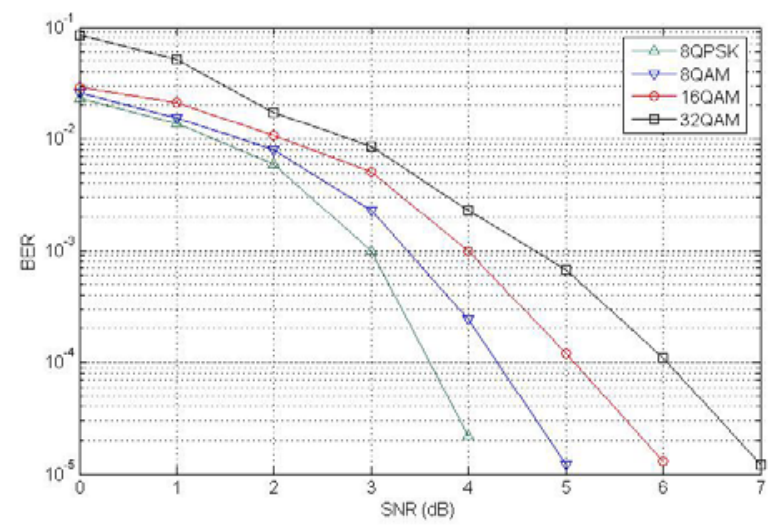

Fig. 3. BER simulation of a concatenated LDPC encoded OFDM system under different modulation schemes in an AWGN channel.

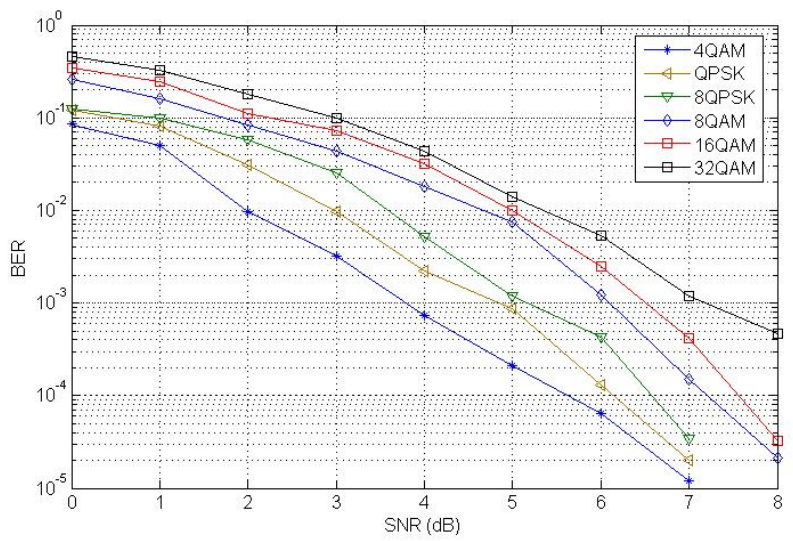

Fig. 4. BER simulation of a concatenated LDPC encoded OFDM system under different modulation schemes in a flat Rayleigh fading channel. 


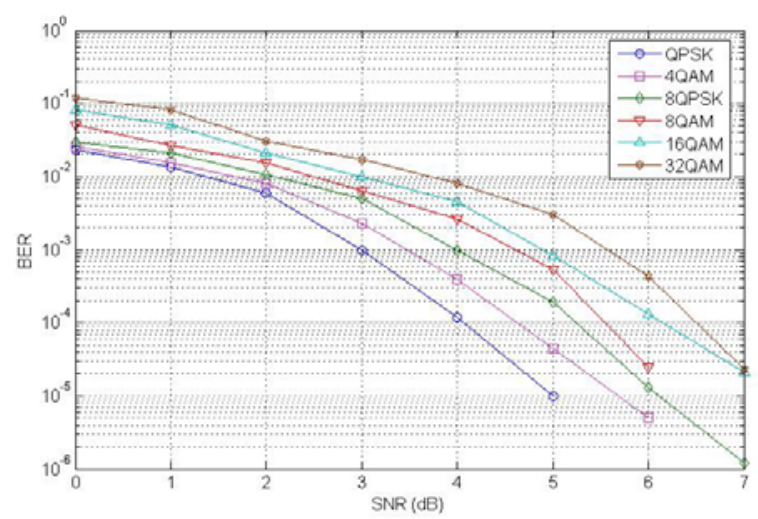

Fig. 5. BER simulation of a concatenated LDPC encoded OFDM system under different modulation schemes in Rician fading channel.

In Fig. 3, the concatenated LDPC-coded OFDM system performances under merely four digital modulations (8PSK, 8QAM, 16QAM and 32QAM) have been shown in terms of BERs as the bit error rates in case of QPSK and QAM are zero at low SNR values and are not shown in Fig. 3. In comparison among four represented graphical illustrations, it is observable that the proposed system provides degraded performance in case of 32QAM. For a typical SNR value of $1 \mathrm{~dB}$, the BER values for 32QAM and 8PSK are 0.0850 and 0.0234 respectively viz. the system performance is improved by $5.60 \mathrm{~dB}$. In Fig. 4, it is observable that the system provides better performance in QAM modulation and degradation of performance occurs in 32QAM on a Raleigh fading channel. In comparison of the QAM with 32QAM modulation, it is found that system performance is improved by $18.24 \mathrm{~dB}$ for a typical SNR value of $5 \mathrm{~dB}$. The BER performance of the concatenated LDPC coded OFDM system on a Rician fading channel is shown in Fig. 5. Due to fading effect, the system performance undergoes significant degradation in 32QAM. Its performance is satisfactory for QAM modulation (Fig. 5). In comparison of the QAM with 32QAM modulation, it is found that the system performance on Rician fading channel is improved by $24.77 \mathrm{~dB}$ for a typical SNR value of $5 \mathrm{~dB}$. Under AWGN and fading channel environment, the transmitted and received color images are shown in

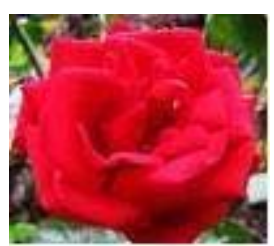

(a)

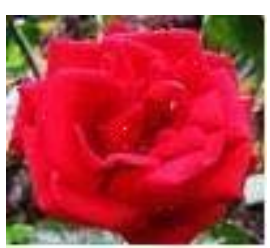

(b)

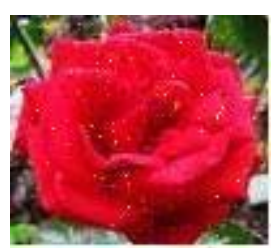

(c)

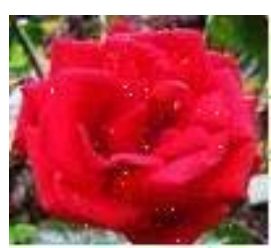

(d)

Fig. 6. Pictorial views of the digital image under various fading channels, (a) transmitted, (b) received in AWGN channel, (c) received in Raleigh fading and (d) received in Rician fading. 


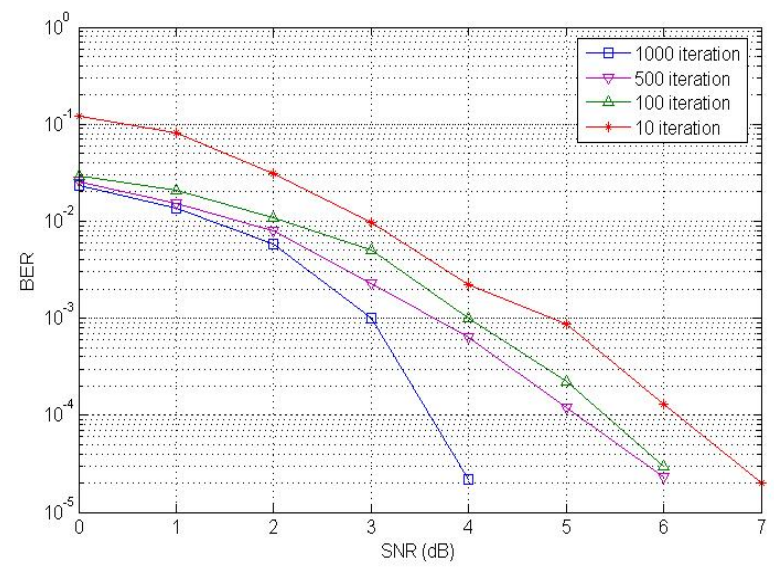

Fig. 7. Impact of the number of iterations on the BER simulation of a concatenated LDPC encoded OFDM system under AWGN channel.

Fig. 6(a) through 6(d) for a typical SNR value of 5dB. As in this paper, iterative decoding algorithm has been used in retrieving transmitted color image; the number of iterations has impact on the bit error rate (Fig. 7). In Fig. 7, it is observable that the system performance is well appreciated at higher number of iterations.

\section{Conclusions}

It can be concluded that the deployment of a concatenated channel coding scheme with low-density parity-check and convolutional codes in orthogonal frequency division multiplexing based wireless communication system under QAM modulation is very much effective in proper identification and retrieval of transmitted digital image in noisy and fading environment. In this paper, we evaluated the performance of a concatenated low density parity check encoded orthogonal frequency division multiplexing system under various digital modulations. We showed that the system achieves good error rate performance on both an AWGN and frequency-selective fading channels.

\section{References}

1. G. L. Stüber, S. W. Mclaughlin, and M. A. Ingram, Broadband MIMO-OFDM wireless communications - Proc. IEEE 92 (2), 271 (2004). doi:10.1109/JPROC.2003.821912

2. W. Zhang, X. -G. Xia and K. B. Letaief, IEEE Wireless Communications, 14 (3), 32 (2007). doi:10.1109/MWC.2007.386610

3. R. G. Gallager, Low density parity check codes, IRE Transaction Information Theory IT -8, 21 (1962). doi:10.1109/TIT.1962.1057683

4. R. G. Gallager, Low Density Parity Check Codes, Research Monograph Series (Cambridge, MIT Press, 1963).

5. M. Chiani, A. Conti, and A. Ventura, IEEE ICC 3, 1183 (2000).

6. D. I. C. MacKay and R. M. Neal, Elec. Lett. 32 (8), 1645 (1996). doi:10.1049/el:19961141 
7. M. Ergen, Mobile Broadband including WiMAX and LTE (Springer Science and Business Media, LLC, USA, 2009).

8. H. Futaki, and T. Ohtsuki, Low-Density Parity-Check (LDPC) Coded OFDM systems, IEEE VTS. $54^{\text {th }}, 1,82$ (2001).

9. Kratochvíl, Tomáš, Radio Engineering, 12 (4), 31 (2003).

10. B. Lu, G. Yue and X. Wang, IEEE Trans. on Signal Processing 52, (2), 348 (2004). doi:10.1109/TSP.2003.820991

11. L. J. Cimini, IEEE Trans. Comm. 33, 665 (1985). doi:10.1109/TCOM.1985.1096357 